\title{
Analysis, Design and Implementation of Quadcopter Control System
}

\section{KyawMyat Thu ${ }^{a}$ and Gavrilov Alexander Igorevich ${ }^{b}$}

\author{
No-5, 2nd Baumanskaya Str., 105005, Moscow, Russian Federation. \\ akyawmyatthu@live.com, balexgavrilov@mail.ru
}

Keywords: Quadcopter, quadrotor, multicopter, Unmanned Aerial Vehicle (UAV), design and implementation, feedback control, altitude control, analysis, modeling.

\begin{abstract}
Quadcopters have generated considerable interest in both the control community due to their complex dynamics and military because of their advantages over regular aerial vehicles. This paper presents the analysis and design of differential equations of the quadcopter dynamics. The behaviour of the dynamic model is examined by simulating the flight of the quadcopter. Stabilization of the quadcopter is conducted by utilizing a PID controller. The PID controller is a simple control method which is easy to implement as the control method of the quadcopter. A simple control method is developed to control the trajectory and altitude of the flight.The proposed dynamical model which comprises gyroscopic effects and its control strategies can be source for future works.
\end{abstract}

\section{Introduction}

Quadcopter, also known as quadrotor, is a helicopter with four rotors. The rotors are directed upwards and they are placed in a square formation with equal distance from the center of mass of the quadcopter. The quadcopter is controlled by adjusting the angular velocities of the rotors, which are spun by electric motors. Quadcopter is a typical design for small unmanned aerial vehicles (UAV) because of the simple structure. Quadcopters are used in surveillance [1], search and rescue [2], construction inspections [3] and several other applications [4].

Current research focusedon complex phenomena of the quadcopter has generated several areas of interest. Dynamical model of the quadcopter is the starting point for all of the studies but more complex aerodynamic properties has been introduced as well. Different control methods have been researched, including several controllers [5], back- stepping control [6], nonlinear $H$ control [8], LQR controllers [7], and nonlinear controllers with nested saturations. Control methods require accurate information from the position and attitude measurements performed with a gyroscope, an accelerometer, and other measuring devices, such asGPS, sonar and laser sensors.

This paper is devoted to modeling of quadcopterdynamics and control system as the basis for further research and development. The first aim is to study the mathematical model of the quadcopter dynamics. The second aim is to develop proper methods for stabilisation and trajectory control of the quadcopter. The challenge is that the quadcopter has six degrees of freedom but there are only four control inputs.

\section{Dynamic Modeling}

A quadcopter is an under actuated aircraft with fixed pitch angle four rotors as shown in Fig. 1. Modeling a vehicle such as a quadcopter is not an easy task because of its complex structure. The aim is to develop a model of the vehicle as realistically as possible.

A typical quadcopter have four rotors with fixed angles and they makequadcopter has four input forces, which are basically the thrust provided by each propellers as shown in Fig. 1. There are two possible configurations for most of quadcopter designs " + " and " $\times$ ". An X-configuration quadcopter is considered to be more stable compared to + configuration, which is a more acrobatic configuration. Propellers 1 and 3 rotates counter clockwise (CW), 2 and 4 rotates counter-counter clockwise (CCW). So that, the quadcopter can maintains forward (backward) motion by increasing (decreasing) speed of 
front (rear) rotors speed while decreasing (increasing) rear (front) rotor speed simultaneously, which means changing the pitch angle. This process is required to compensate the action/reaction effect (Third Newton's Law). Propellers 1 and 3 have opposite pitch with respect to 2 and 4, so all thrusts have the same direction.
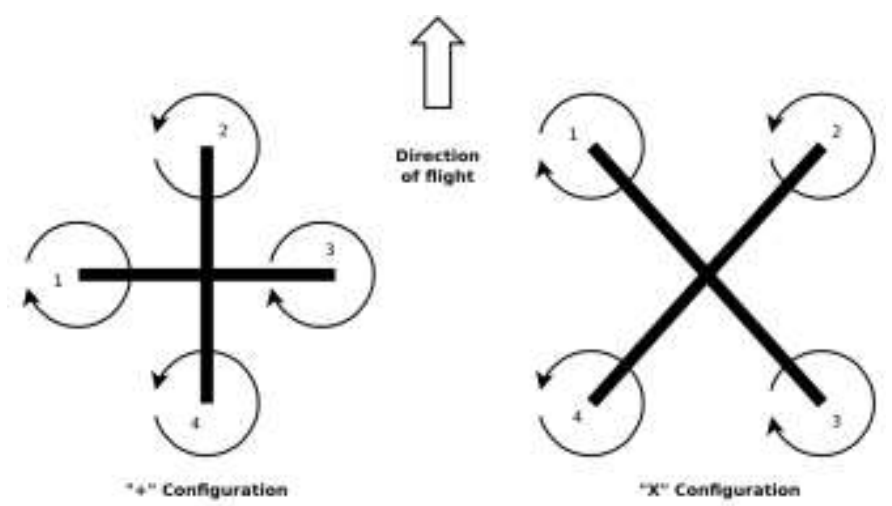

Fig. 1. Twomai types of quadrocopterconfiguration.

There are two reference systems that have to be defined as a reference which are Inertial reference system (Earth frame- $X E, Y E, Z E$ ) and quadrotorreference system (Body frame- $X B, Y B, Z B$ ). The reference system frames are shown in Fig. 2. The dynamics of quadcopter can be describe in many different ways such as quaternion, Euler angle and direction matrix. However, in designing attitude stabilization control reference in axis angle is needed, so the designed controller can achieve a stable flight. In attitude stabilization control, all angle references in each axis must be approximately zero especially when take-off, landing or hover. It ensures that, the quadcopter body always is in horizontal state, when external forces are applied on it. The quadcopter orientation can be defined by three Euler angles which are roll angle $(\Phi)$, pitch angle $(\theta)$ and yaw angle $(\varphi)$.
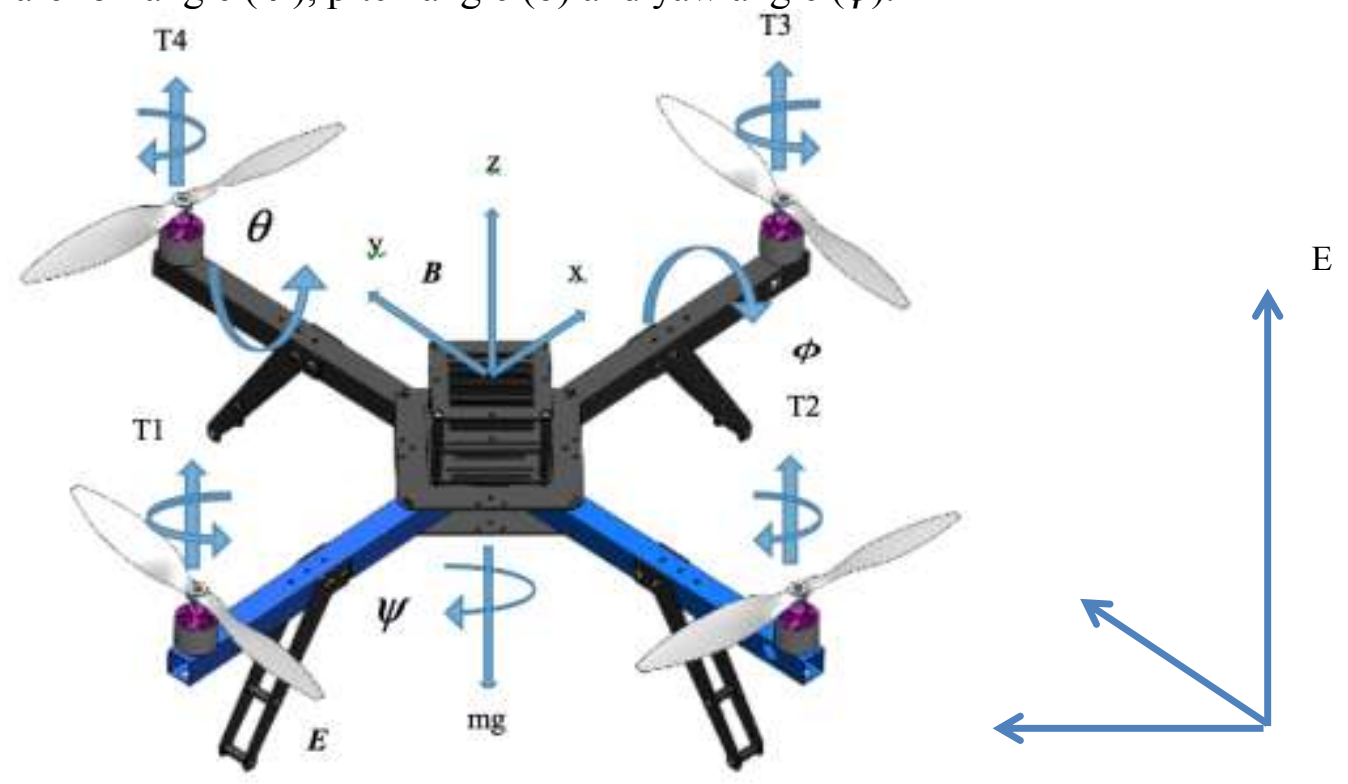

Fig. 2. Forces, moments and reference systems(body frames) of a quadcopter.

where,

$\omega_{1}, \omega_{2}, \omega_{3}, \omega_{4}$ : Rotation speeds (angular velocity) of the propellers

$T_{1}, T_{2}, T_{3}, T_{4}$ : Forces generated by the propellers

$F_{i} \propto \omega_{i}^{2}$ : On the basis of propeller shape, air density, etc.

$m$ : Mass of the quadcopter

$m g$ : Weight of the quadcopter

$\phi, \theta, \psi:$ Roll, pitch and yaw angels

The position of the quadcopter is defined in the inertial frame $x, y, z$-axes with $\xi$. The attitude, i.e. the angular position, is defined in the inertial frame with three Euler angles $\eta$. Pitch angle $\theta$ 
determines the rotation of the quadcopter around the y-axis. Roll angle $\varphi$ determines the rotation around the $\mathrm{x}$-axis and yaw angle $\psi$ around the z-axis. Vector $q$ contains the linear and angular position vectors

$$
\xi=\left[\begin{array}{l}
x \\
y \\
z
\end{array}\right], \quad \eta=\left[\begin{array}{l}
\phi \\
\theta \\
\psi
\end{array}\right], \quad q=\left[\begin{array}{l}
\xi \\
\eta
\end{array}\right] .
$$

The origin of the body reference (body frame) is in the center of mass of the quadcopter. In the body frame, the linear velocities are determined by $J B$ and the angular velocities by $\omega$.

$$
J B=\left[\begin{array}{l}
J x, B \\
J y, B \\
J z, B
\end{array}\right], \quad \omega=\left[\begin{array}{l}
p \\
q \\
r
\end{array}\right] .
$$

The rotation matrix from the body frame to the inertial frame is

$$
R=\left(\begin{array}{ccc}
c_{\psi} c_{\theta} & c_{\psi} s_{\theta} s_{\phi}-s_{\psi} c_{\phi} & c_{\psi} s_{\theta} c_{\phi}+s_{\psi} s_{\phi} \\
s_{\psi} c_{\theta} & s_{\psi} s_{\theta} s_{\phi}+c_{\psi} c_{\phi} & s_{\psi} s_{\theta} c_{\phi}-c_{\psi} s_{\phi} \\
-s_{\theta} & c_{\theta} s_{\phi} & c_{\theta} c_{\phi}
\end{array}\right)
$$

in which $S_{x}=\sin (x)$ and $C_{x}=\cos (x)$. The rotation matrix $R$ is orthogonal thus $R^{-1}=R^{T}$ which is the rotation matrix from the inertial frame to the body frame. time,

There are 3 types of angular speeds which can describe as the derivative of $(\varphi, \theta, \psi)$ with respect to

$$
\stackrel{\circ}{\phi}=\text { Roll rate, } \stackrel{\circ}{\theta}=\text { Pitch rate, } \stackrel{\circ}{\psi}=\text { Yaw rate. }
$$

Considering the hovering condition of quadcopter gives 4 equations of forces, directions, moments and rotation speeds. Those are describe by following,

Equilibrium of forces : $\sum_{i}^{4}=1 T_{i}=-m g$

Equilibrium of directions: $T_{1,2,3,4} \| g$

Equilibrium of moments: $\sum_{i}^{4}=1 M_{i}=0$

Equilibrium of rotation speeds: $\left(\omega_{1}+\omega_{3}\right)-\left(\omega_{2}+\omega_{4}\right)=0$,

And the consequence is: $\stackrel{\circ}{\phi}=0, \stackrel{\circ}{\theta}=0, \stackrel{\circ}{\psi}=0$.

By increasing/decreasing the rotation speed of all the propellers, the quadcopter can make movements flying up and down,

Flying up: $\sum_{i}^{4}=1 T_{i}>-m g$, Flying down: $\sum_{i}^{4}=1 T_{i}<-m g$, Euler angles and rates remain 0 .

Changing the equilibrium of propellers speed, directions and moments gives the following equations of yaw, roll and pitch of quadcopter.

$$
\begin{array}{ll}
\text { Yaw: } \dot{\psi}=k_{Y}\left(\left(\omega_{1}+\omega_{3}\right)-\left(\omega_{2}+\omega_{4}\right)\right) & \phi=\int \dot{\psi} d t \\
\text { Roll: } \stackrel{\circ}{\phi}=k_{R}\left(\left(\omega_{1}+\omega_{4}\right)-\left(\omega_{2}+\omega_{3}\right)\right) & \phi=\int \dot{\phi} d t \\
\text { Pitch: } \stackrel{\circ}{\theta}=k_{P}\left(\left(\omega_{1}+\omega_{2}\right)-\left(\omega_{3}+\omega_{4}\right)\right) & \theta=\int \dot{\theta} d t
\end{array}
$$

Thus, decreasing the 2 nd rotor velocity and increasing the 4 th rotor velocity acquire the roll movement. Similarly, decreasing the 1 st rotor velocity and increasing the 3 th rotor velocity acquire 
the pitch movement. Increasing the angular velocities of two opposite rotors and decreasing the velocities of the other two acquire yaw movement.

\section{Equation of Movement}

Assume a common factor of proportionality $k$ and $F=\sqrt{T}$, each equation of movement for quadcopterhas written down below:

$$
\begin{gathered}
\dot{\phi}=k\left(\left(\omega_{1}+\omega_{4}\right)-\left(\omega_{2}+\omega_{3}\right)\right)=k \omega_{1}-k \omega_{2}-k \omega_{3}+k \omega_{4} \\
\dot{\theta}=k\left(\left(\omega_{1}+\omega_{2}\right)-\left(\omega_{3}+\omega_{4}\right)\right)=k \omega_{1}+k \omega_{2}-k \omega_{3}-k \omega_{4} \\
\dot{\psi}=k\left(\left(\omega_{1}+\omega_{3}\right)-\left(\omega_{2}+\omega_{4}\right)\right)=k \omega_{1}-k \omega_{2}+k \omega_{3}-k \omega_{4} \\
F=k\left(\left(\omega_{1}+\omega_{2}+\omega_{3}+\omega_{4}\right)\right)=k \omega_{1}+k \omega_{2}+k \omega_{3}+k \omega_{4}
\end{gathered}
$$

by using matrices:

$$
\begin{gathered}
\left(\begin{array}{c}
a \\
\phi \\
c \\
\theta \\
\vdots \\
\psi \\
F
\end{array}\right)=\left(\begin{array}{cccc}
k & -k & -k & k \\
k & k & -k & -k \\
k & -k & k & -k \\
k & k & k & k
\end{array}\right)\left(\begin{array}{l}
\omega_{1} \\
\omega_{2} \\
\omega_{3} \\
\omega_{4}
\end{array}\right) \\
\left(\begin{array}{c}
a \\
\phi \\
\theta \\
\sigma \\
\psi \\
F
\end{array}\right)=\left(\begin{array}{cccc}
k & -k & -k & k \\
k & k & -k & -k \\
k & -k & k & -k \\
k & k & k & k
\end{array}\right)\left(\begin{array}{l}
\omega_{1} \\
\omega_{2} \\
\omega_{3} \\
\omega_{4}
\end{array}\right)=K\left(\begin{array}{l}
\omega_{1} \\
\omega_{2} \\
\omega_{3} \\
\omega_{4}
\end{array}\right)
\end{gathered}
$$

According to equation (8), controlling the four input forces (roll, pitch, yaw, thrust) can be write down as below,

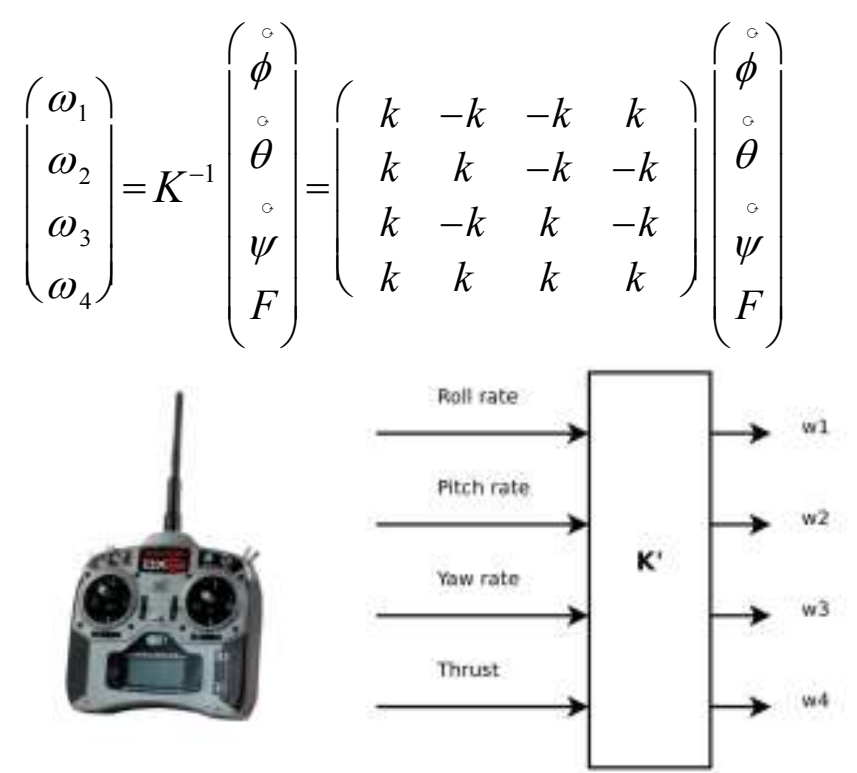

Fig. 3. Controlling the Roll, Pitch, Yaw and Total Thrust Force. 
To control the trajectory and altitude of quadcopter needs to measure the variables through a sensor (e.g. 3-axis gyroscope, accelerometer,..etc.), compare the measure value with desired set point and apply the correction to the system on the basis of the error. It should be work no matter the components and it should be work in the presence of uncontrollable variables, e.g. air density, wind, etc.

Therefore, the PID controller has been developed to control the trajectory and altitude of the flight and for more simplercontrol algorithm, which is easy to implement as the control method of the quadcopter.

PID function and controller:

$$
C:=x x x \_ \text {rate_controller }(e)
$$

That is:

$$
c(t):=K_{p} e(t)+K_{i} \int_{0}^{t} e(\tau) d(\tau)+K_{d} \frac{d e(t)}{d t}
$$

In a discrete world (at $k^{\text {th }}$ sampling instant):

$$
C(k):=K_{p} e(k)+K_{i} \sum_{j=0}^{k} e(j) \Delta T+K_{d} \frac{e(k)-e(k-1)}{\Delta T}
$$

where constants $K_{p}, K_{i}, K_{d}$ define the behavior of the controller:

- $K_{p}$ drives the short-term action;

- $K_{i}$ drives the long-term action;

- $K_{d}$ drives the action on the basis of the "error trend".

Constants $K_{p}, K_{i}, K_{d}$ are estimated by using Ziegler-Nichols tuning method [9].

The basic and full feedback control block diagram is shown in Fig. 4, 5. In these diagrams the inputs to the PID controller are the rotor speed error signals, and derivative of the error signal which corresponds to the PID variables.

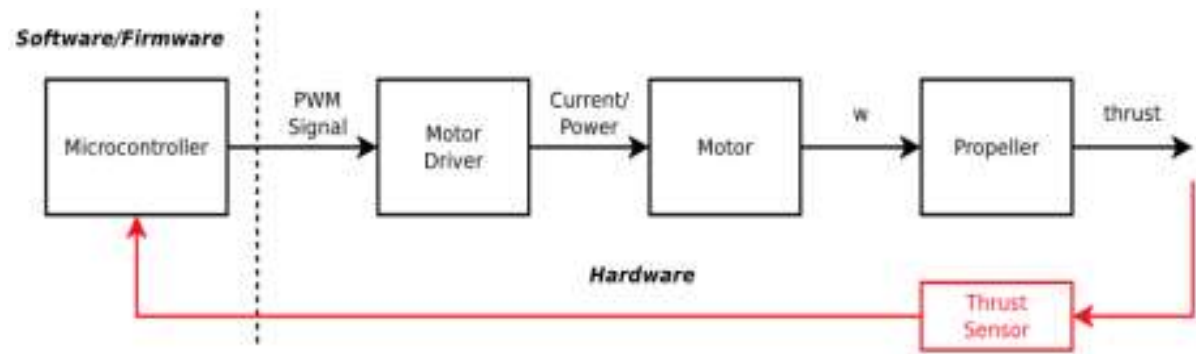

Fig. 4. The basic block diagram of the feedback control system of quadcopter.

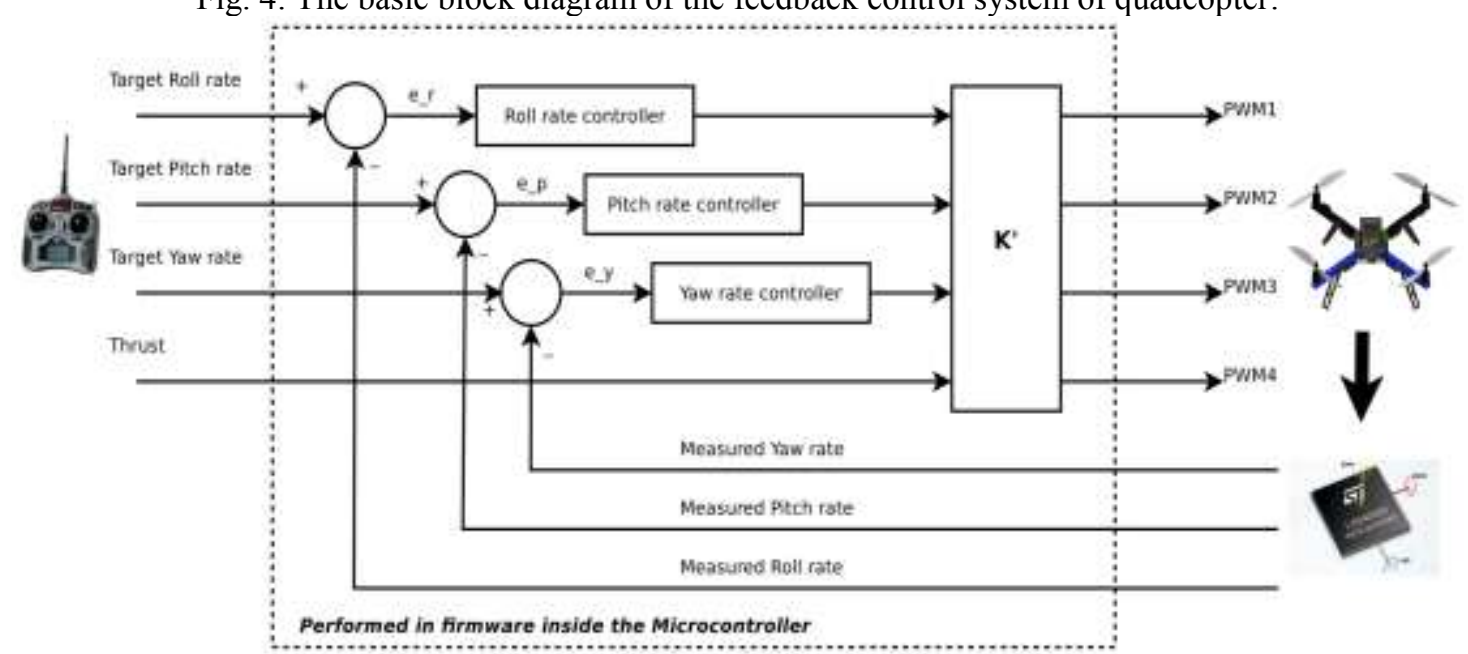

Fig. 5. Full block diagram of the feedback control system of quadcopter. 
On the other hand, the feedback control system can be algorithmically described as following,

\section{Simulation}

The mathematical model of the quadcopter is implemented for simulation in Matlab 2013 with Matlab programming language. Parameter values from [3] are used in the simulations and are presented in Table 1.

Table 1. Parameters of the system

\begin{tabular}{|c|c|c|c|}
\hline Symbol & Description & Value & Unit \\
\hline$g$ & Weight of the quadcopter & 9.81 & {$\left[\mathrm{~m} / \mathrm{s}^{2}\right]$} \\
\hline$m$ & Mass of the quadcopter & 0.75 & {$[\mathrm{~kg}]$} \\
\hline$l$ & Distance from center to motor & 0.26 & {$[\mathrm{~m}]$} \\
\hline$J x$ & Moment of inertia about $x$ axis & 0.019688 & {$\left[\mathrm{kgm}^{2}\right]$} \\
\hline$J y$ & Moment of inertia about $y$ axis & 0.019688 & {$\left[\mathrm{kgm}^{2}\right]$} \\
\hline$J z$ & Moment of inertia about $z$ axis & 0.03938 & {$\left[\mathrm{Ns}^{2}\right]$} \\
\hline $\mathrm{Kt}$ & Propeller Force Constant & $3.13 \times 10^{-5}$ & {$\left[\mathrm{Ns}^{2}\right]$} \\
\hline $\mathrm{Kq}$ & Propeller Torque Constant & $7.5 \times 10^{-7}$ & \\
\hline
\end{tabular}

Table 2. Parameters of the PID controller

\begin{tabular}{|c|c|c|c|}
\hline \multirow{2}{*}{$\begin{array}{c}\text { Variable } \\
i\end{array}$} & \multicolumn{3}{|c|}{ Parameter Value } \\
\cline { 2 - 4 } & $K_{i, P}$ & $K_{i, D}$ & $K_{i, I}$ \\
\hline$x$ & 1.85 & 0.75 & 1.00 \\
\hline$y$ & 1.75 & 0.75 & 1.00 \\
\hline$z$ & 1.85 & 0.75 & -00 \\
\hline$\phi$ & 3.00 & 0.75 & - \\
\hline$\theta$ & 3.00 & 0.75 & - \\
\hline$\psi$ & 3.00 & 0.75 & - \\
\hline
\end{tabular}

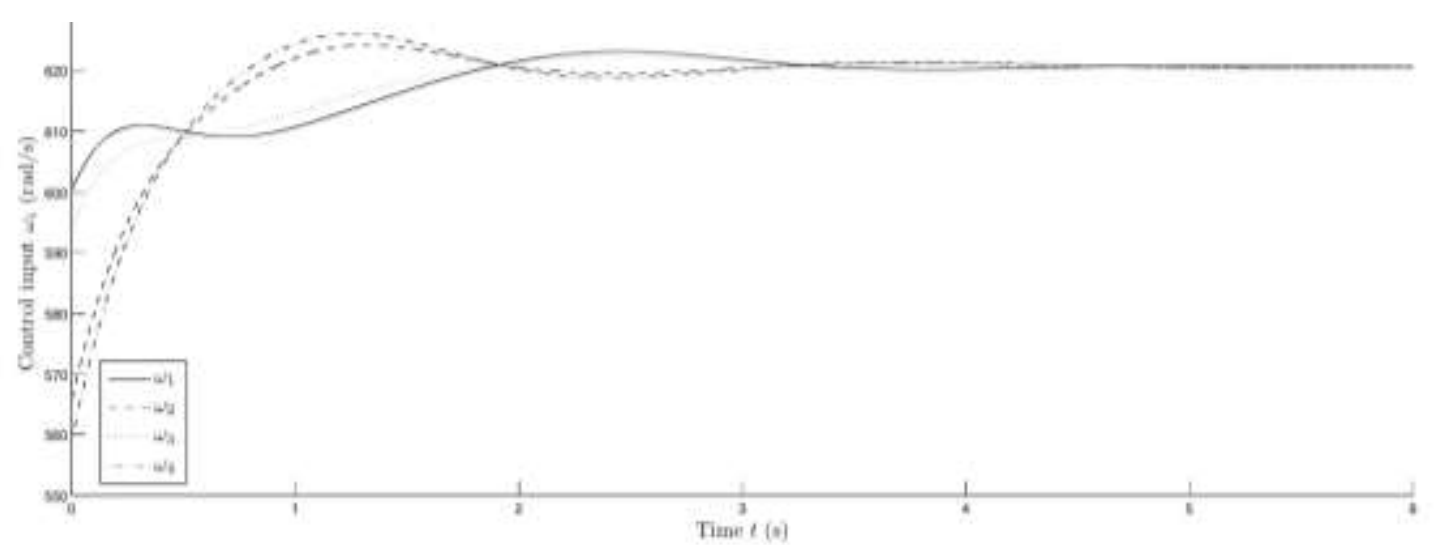

Fig. 6. Control inputs $\omega_{i}$

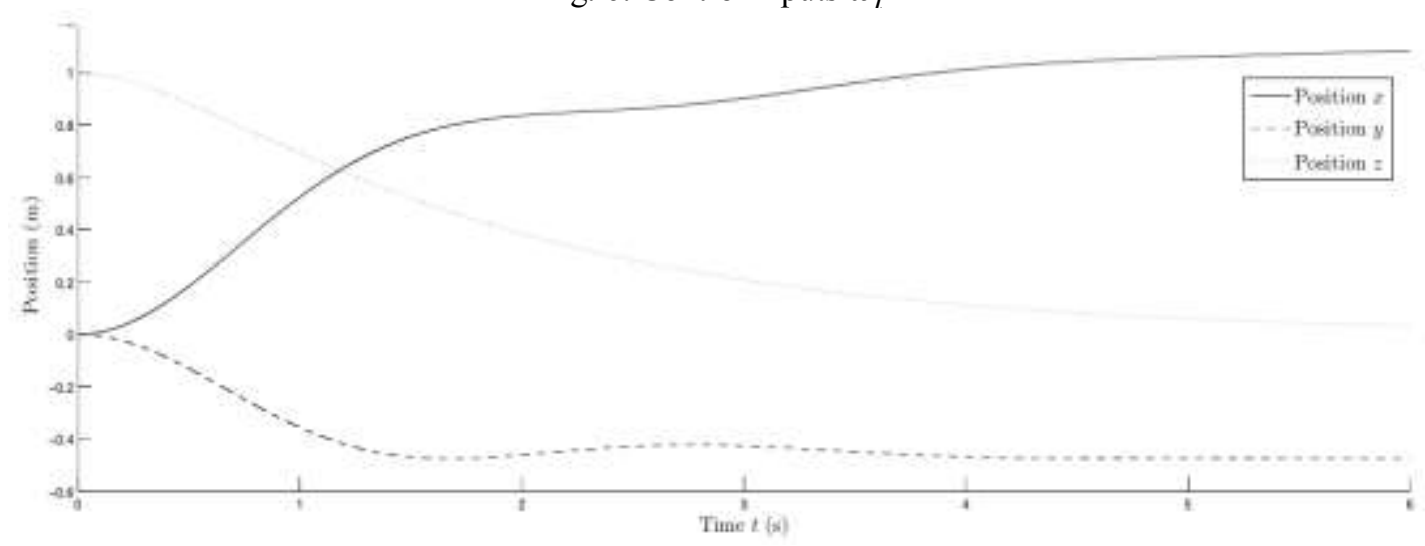

Fig. 7. Positions $x, y$, and $z$.

Control inputs $\omega_{i}$, positions $\xi$ and the angles $\eta$ during the simulation are presented in Fig. 6, 7, and 8. The altitude and the angles are stabilized to zero value after 5 seconds. The position of the quadcopter is close to the planned position after 4 seconds but the position keeps fluctuating close to the planned values for several seconds. The angles variate greatly during the simulation to achieve the 
wanted positions, velocities, and accelerations. The values of the control inputs oscillated during the acceleration but then their behavior became more stable.

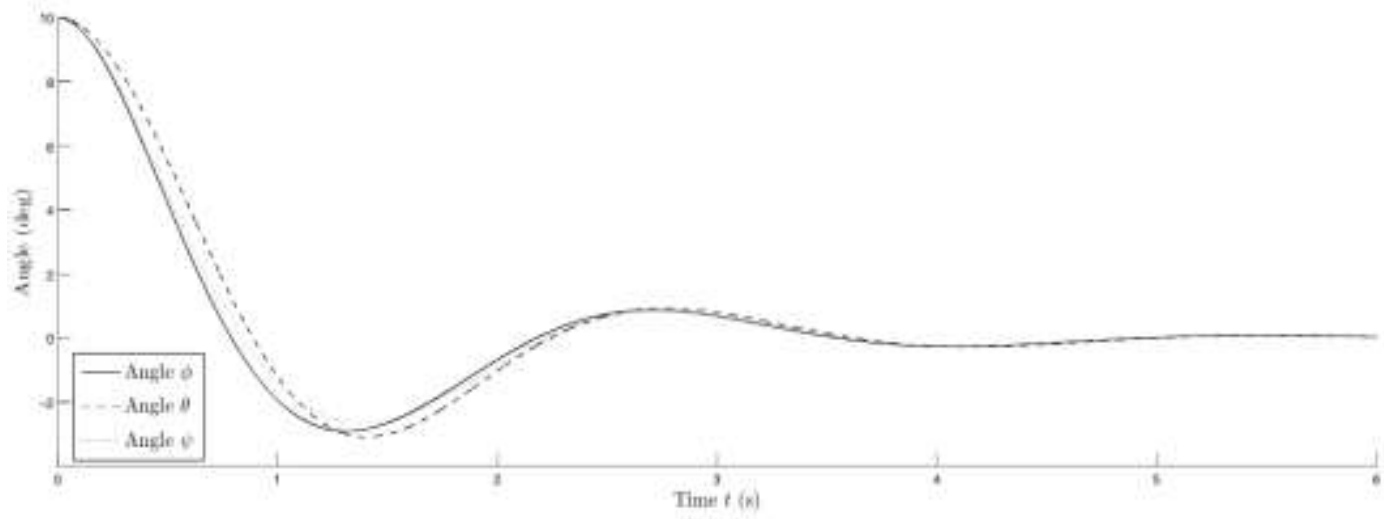

Fig. 8. Angels $\phi, \theta, \psi$.

\section{Conclusion}

This paper studied analysis, mathematical modeling and control of a quadcopter. The mathematical model of quadcopter dynamics was presented and the differential equations were implemented into real time simulation model. The model was verified by simulating the flight of a quadcopter with Matlab.The simulation proved the presented mathematical model to be realistic in modeling the position and attitude of the quadcopter. Simulation results also showed that the PID controller was efficient in stabilizing the quadcopter to the desired altitude and trajectory.

\section{References}

[1] R. Vezzani, R. Cucchiara, A. Malizia, L. Cinque, "3-D Virtual Environments on Mobile Devices for Remote Surveillance", IEEE International Conference on Video and Signal Based Surveillance, (November 2006), p.100.

[2] H.A.F. Almurib, P.T. Nathan, T.N. Kumar, "Control and path planning of quadrotor aerial vehicles for search and rescue”, SICE Annual Conference (SICE), ( 2011), pp. 700-705.

[3] S. Emelianov, A. Bulgakow, D. Sayfeddine, "Aerial Laser Inspection of Buildings Facades Using Quadrotor”, Procedia Engineering, Volume-85, (2014), pp. 140-146.

[4] J. Willmann, F. Augugliaro, T. Cadalbert, R. D’Andrea, F. Gramazio, M. Kohler, “Aerial Robotic Construction: Towards a New Field of Architectural Research", International journal of architectural computing, Vol. 10-3, (2012), pp.439-460.

[5] A. I. Gavrilov, KyawMyat Thu, E. A.Budnikova, "Synthesis of automatic control system quadrocopter", International Conference on Control in Marine and Aerospace System,UMAS-2014, (Sep. 2014).

[6] T. Madani and A. Benallegue, "Backstepping control for a quadrotorheli- copter", IEEE/RSJ International Conference on Intelligent Robots and Sys- tems, (2006), pp. 3255-3260

[7] G. V. Raffo, M. G. Ortega, and F. R. Rubio, “An integral predictive/nonlinear $H_{\square}$ control structure for a quadrotor helicopter", Automatica, vol. 46, no. 1, (2010), pp. 29 - 39.

[8] P. Castillo, R. Lozano, and A. Dzul, "Stabilisation of a mini rotorcraft with four rotors, IEEE Control Systems Magazine”, (Dec.2005), pp. 45-55.

[9] Information on http://www.engr.mun.ca/ nick/eng5951/Ziegler_Nichols.pdf 